-Gel“. Das Plasma der wachsenden und ausgewachsenen Zellen befindet sích nicht mehr in vorwiegend gelatineartigem Zustand, es ist viel reicher an Wasser und als "Sol“ zu bezeichnen.

Diese Umwandlung des Plasmas von dem Zustande des Gels in den eines Sols ist, wie ich glaube, der wichtigste Moment des Wachstums. Dieser Hydratationsproze ${ }^{3}$ des Plasmas ist keine einfache Quellung. Die, wenn auch bedeutende Wassermenge, die in das Plasma dringt, verbindet sich zweifellos mit dem Eiweibmolekäl. Das bestätigen auch die Versuche Wo.Pa uli's und seiner Schüler mit Eiweil in vitro sowie die Irreversibilität des Solzustandes, wenigstens in den Grenzen unserer Versuche, allein nicht in der Natur, wo sich diese Vorgänge abspielen. Es ist begreiflich, daB durch Beschleunigung des Hydratationsprozesses der Plasmakolloide auch das Wachgtum beschleunigt wird, denn dadurch werden die Umstände ermöglicht, welche die Umwandlung des Plasmas aus dem Gelznstande in den des Sols: beschleunigen und ferner wird die Beschleunigung der Quellung des ubrigen gelatineartigen Plastmabestandteiles beschleunigt.

Der osmotlsche Druck des Zellsaftes und das Wachstum der Zellhaut haben für die Wachstumsmechanik weitaus nicht eine derartige Bedentung, wie man sie ihnen früher zuschrieb.

Es gibt in der botanischen Literatur Beispiele, die darauf schlieben lassen, dat die Neubildung der Zellulose aus dem Plasma, bei den Hydratations- sowohl wie bei den Dehydratationsprozessen, erfolgen kann.

Das weitere Schicksal der neugebildeten Zellulose hängt von dem Plasma ab. Wenn das Plasma sich im Hydratatlonszustande befindet, wird die Zellulose zum Flachenwachstum der Zellhaut verbraucht, bei der Hemmung oder Beendigung dieses Prozesses wird dagegen die sich neubildende Zellulose für das Dickenwachstum der Zellwand verbraucht.

Die Rolle des Turgors besteht vor allem in der Vergrößerung der Obetfläche des Plasmas und der VergröBerung des Zellvolumens; es ist aber zu bemerken, dab dieselbe Vergrößerung des Plasmaumfanges, das Ausdehnen desselben nur dann erfolgt, wenn sich das Plasma hierfür im richtigen Zustande befindet, $d$. h. wenn darin der Hydratationsprozef der Kolloide vor sich gehen kann, da im entgegengesetzten Falle die mechanische Koagulation des Plasmas zu erwarten wäre.

Wenn man deswegen bei den Wachstumserscheinungen der wachsenden Zelle vor allem mit den Aenderungen des Charakters des Plasmas zu rechnen hat und nicht mit der Größe des Turgordruckes und den Eigenschaften der Zellhaut, so haben wir dann folgende drei hochwichtige Zustände des Plasmas während des Wachstums festzustellen:

1. Die feste Phase des Plasmas, in welchem Zustande wir es in den Samen und Sporen finden; 2. die Gel-Phase (nach J. Sachs' Embryonalphase), in welchen Zustand das Plasma aus der ersten Phase übergeht; 3 . die Sol-Phase (Streckungsphase nach $\mathrm{Sachs}$ ), in welchen Zustand das Plasma aus der zweiten Phase übergeht und in dem wir es gewöhnlich in der Zelle vorfinden.

Die Periode des Wachstums, welche jede Zelle in ihrer Entwickelung durchmachen muB, kann durch die ungleiche Schnelligkeit des verlaufenden Hydratations - Prozesses der Zellenkolloide erklärt werden.

Botanisches Laboratorium der böhmischen Universität Prag.

\title{
Ueber den Einfluß des Lösungsmittels auf die Viskosität von Kautschuklösungen.
}

Von F. Kirchh of (Steyr, Ob.-Oest.)

(Eingegangen am 6. April 1914)

Auf die grobe Bedeutung der Viskosität als Mittel zur Erforschung des kolloiden Zustandes wurde vom Herausgeber dieser Zeitschrift wiederholt hingewiesen ${ }^{1}$ ). Aach die zahlreichen dies-

2) Wo. Ostwald, Ueber die Bedeutung der Viskosittit für das Studium des kolloiden Zustandes. Vortrag, gehalten a. d. Meeting d. Faraday Soc. am 12. Marz 1913; Koll.-Zeitschr. 12, 213 (1913), Faraday. Heft. bezüglichen Publikationen in derselben zeigen deutlich, das ihr bereits von vielen Forschern gebührende Aufmerksamkeit geschenkt wird.

Besonders auf den Gebiete der Kautschukchemie hat die Viskositătsmessung schon recht interessante Erscheinungen aufgedeckt, welche durch andere Mittel kaum gefunden worden wären.- Auch scheint die Bestimmung der Viskosität berufen zu sein, ein wichtiges Kri- 
terium bei der Bewertung des Kautschuks zu werden. Um eine solche Wertbestimmung aus der Viskosität einwandfrei herleiten zu können, ist es notwendig, den Einflub jedes einzelnen in Betracht kommenden Faktors möglichst genau zu kennen. Ein solcher Faktor, der meines Wissens noch keine Berücksichtigung gefunden, ist der Einflub der Natur des Losungsmittels auf die Viskosität der damit hergestellten Kautschuklösungen.

Ebenso wichtig wie die Erkenntnis von der Bedeutung der Viskosität ist die Feststellung einer möglichst einwandfreien und allgemeinen Methode ihrer Messung. Von G. Fol ${ }^{2}$ ) wurde vor Jahresfrist an dieser Stelle eine Zusammenstellung der im Laufe der Zeit vorgeschlagenen Methoden und eine kritische Beurteilung derselben gegeben. G. Fol gab hierbei dem von Ph. Schidrowitz und A. H. Golds brough benutzten $\mathrm{Os}$ twa I d'schen Kapillar-Viskosimeter vor allen anderen Apparaten den Vorzug und machte auf Grund eingehender eigener Untersuchungen Vorschläge über die wünschenswerteste Art von Viskositätsbestimmungen.

Auf dem Meeting der Faraday Society, London 1913, machte E. Hatschek ${ }^{3}$ ) auf einen modifizierten von $E$. Couetie angegebenen Apparat aufmerksam, welcher Aenderungen in der Schergeschwindigkeit bei den Viskositätsmessungen gestatte. In einer späteren Abhandlung () wies derselbe Forscher auf den Unterschied der Viskositätswerte, die mit dem Couette'schen Apparat und mit dem Ost: wald-Viskosimeter erhalten wurden, hin. Die Ursache sei in dem zu hohen Geschwindigkeits-

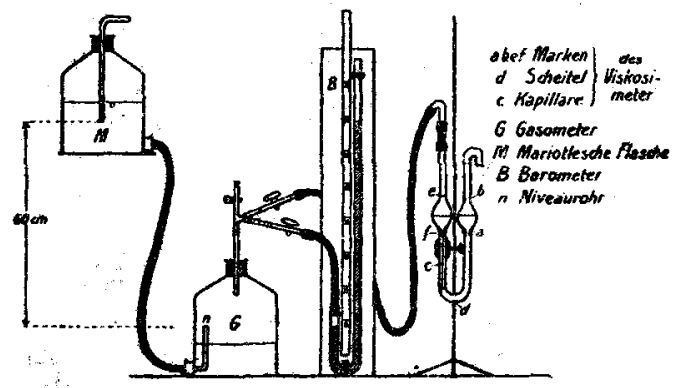

Fig. 1

2) G. F o l, Viskositătsbest. an Kautschuklösungen. Koll.-Zeitschr. 12, 131 (1913).

8) E. Hatschek, Die allgemeine Theorie der Viskositat zweiphasiger Systeme. Koll.-Zeitschr. 12, 238 (1913).

9) Derselbe, Die Viskosităt d. Emulsoid-Sole und ihre Abhängigkeit von der Schergeschwindigkeit. Koll.Zeitschr. 13,88 (1913). gefälle im Kapillar-Viskosimeter zu suchen, welches auf labile Sole eine zerstörende Wirkung ausüben könnte. Trotz dieser Bedenken dürfte dern Kapillar-Viskosimeter wegen seiner weit größeren Einfachheit und Allgemeinheit der Vorzug vor dem theoretisch gewiß einwandfreieren Couette'schen Apparat zu geben sein.

Versuche über die Abhängigkeit der Viskositat von der Durchflub. geschwindigkeit im KapillarViskosimeter.

$\mathrm{Zu}$ den folgenden Versuchen wurde ein von L. Ubbelohde modifiziertes Ostwaldsches Kapillar-Viskosimeter verwendet. Dieses bietet den Vorteil, dab durch die symmetrische Anordnung der Kugeln der EinfluB des spezifischen Gewichtes des Lösungsmittels eliminiert wird. Die Abmessungen des Instrumentes (Fig. 1) waren die folgenden:

Volumen zwischen den Marken a, b: $6,09 \mathrm{ccm}$, Querschnitt der Kapillare: $0,403 \mathrm{qmm}$,

Radius der Kapillare: 0,356 $\mathrm{mm}$,

Ausflußzeit für $6,09 \mathrm{~cm}$ Wasser bei $18^{\circ}$ : 10,0 Sekunden,

Ausgeflossenes Volumen pro Sekunde : $0,609 \mathrm{ccm}$.

Die Dimensionen der Kapillare wurden durch Wägung eines Quecksilberfadens von bekannter Länge, der in ihr erzeugt wurde, ermittelt. In analoger Weise das Volumen der Kugel. Bei Beginn der Versuche reichte die Flüssigkeit von Marke e bis nahe an Marke a, was durch Ansaugen bis zum Scheitel d erreicht wurde.

Um den Einfluß der Durchflutgeschwindigkeit auf die Viskosität zu studieren, wurde das Viskosimeter mit einem unter konstantem Druck stehenden Gasometer in Verbindung gebracht. Der Druck konnte mit Hilfe einer Mariotte schen Flasche konstant gehalten und durch Aenderung der Höhe der Wassersăule variiert werden. Ein Barometerrohr diente zur Messung der Wassersäule. Die Temperatur war innerhalb enger Grenzen konstant und betrug durchschnittlich $20^{\circ} \mathrm{C}$. Die gesamte Anordnung gibt Fig. I wieder.

Durch die folgenden Versuche wurden die Durchflubzeiten der bei den weiteren Messungen verwendeten Lösungsmittel unter wechselndem Druck bestimmt. 
Tabelle I

\begin{tabular}{|c|c|c|c|c|}
\hline \multirow{2}{*}{$\begin{array}{c}\text { Druck in } \\
\text { Wasser } \\
\mathrm{cm}\end{array}$} & \multicolumn{4}{|c|}{ Durchflußzeiten $r$ in Sekunden } \\
\hline & $\begin{array}{c}\text { Wasser } \\
\text { dest. }\end{array}$ & Benzin & Benzol & $\begin{array}{l}\text { Pentiachtor- } \\
\text { Lithan }\end{array}$ \\
\hline & & & 28.6 & --- \\
\hline & & & 15 & 54,4 \\
\hline & 18 , & 13 & 11,6 & 35,6 \\
\hline 10 & & 10, & & 27,2 \\
\hline & & 9,0 & 7,8 & 22,0 \\
\hline 60 & 10,0 & 7,6 & 6,8 & 19,2 \\
\hline
\end{tabular}

Alle übrigen hier benutzten Flüssigkeiten ordnen sich zwischen Benzol und Pentachloräthan ein. Aus den Versuchen ergibt sich, wie nicht anders zu erwarten war, eine deutliche Abhängigkeit der Durchflubzeiten vom spezifischen Gewicht der Flüssigkeiten.

Tabelle II

\begin{tabular}{|c|c|c|}
\hline Flitssigkeit & $\begin{array}{c}\text { Spez. } \\
\text { Gewicht }\end{array}$ & $\begin{array}{l}\text { Durchflubzeiten } \tau_{60} \\
\text { (bei } 60 \mathrm{~cm} \text { Druck } \\
\text { und } 18^{\circ} \mathrm{C} \text { ) in } \\
\text { Sekunden }\end{array}$ \\
\hline $\begin{array}{l}\text { Wasser } \\
\text { Benzin : }: \text { : } \\
\text { Benzol } \\
\text { Trichlorathylen } \\
\text { Tetrachlorathan } \\
\text { Tetrachlorkohlenstoff } \\
\text { Pentachloräthan . }\end{array}$ & $\begin{array}{l}1,00 \\
0,74 \\
0,87 \\
1,47 \\
1,60 \\
1,63 \\
1,70\end{array}$ & $\begin{array}{r}10,0 \\
6,8 \\
7,6 \\
9,0 \\
15,2 \\
11,2 \\
19,2\end{array}$ \\
\hline
\end{tabular}

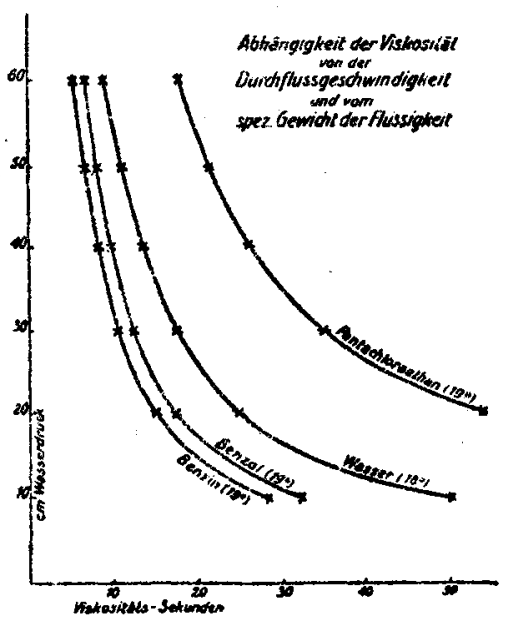

Fig. 2

Trägt man die in Tab. I zusammengestellten Werte in ein Diagramm ein (Fig. 2), so sieht man, daß von ungefähr $60 \mathrm{~cm}$ Druck angefangen, die Durchflubzeiten für ein und dieselbe Flüssigkeit vom Druck praktisch unabhängig werden. In der Folge wurde daher dieser Druck alienViskositätsmessungen zugrunde gelegt, da auch die Viskositäts-Koeffizienter? der Kautschuklösungen bis zu 1 Proz. Konzentration bei diesem Druck von der Durchflubgeschwindigkeit unabhängig werden. Tab. III gibt eine Uehersicht der Abhängigkeit der Viskosität von der Kautschukkonzentration und der Durchfluligeschwindigkeit. Die letzten zwei Vlumnen zeigen bereits den Einflub des Lösungsmittels. In der graphischen Darstellung (Fig. 3) würden sich die Viskositätskurven der übrigen einprozentigen Lösungen (mit Ausnahme der in Benzin) zwischen die der Benzolund der Pentachloräthanlösung einordnen.

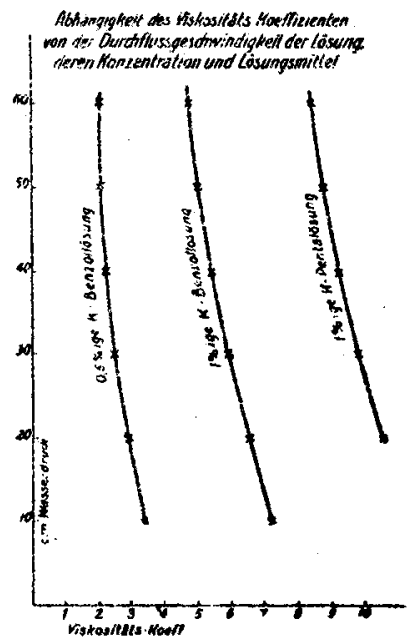

Fig. 3

Tabelle III

\begin{tabular}{|c|c|c|c|c|}
\hline $\begin{array}{l}\text { Druck in } \\
\text { Wasser- } \\
\text { säule } \\
\mathrm{cm}\end{array}$ & \multicolumn{4}{|c|}{ 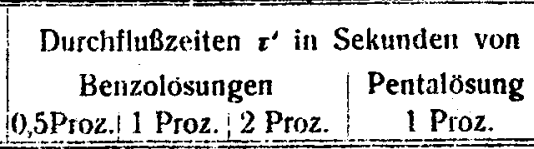 } \\
\hline $\begin{array}{l}10 \\
20 \\
30 \\
40 \\
50 \\
60\end{array}$ & $\begin{array}{r}107 \\
50 \\
33 \\
24 \\
19 \\
16\end{array}$ & $\begin{array}{r}231 \\
115 \\
75 \\
59 \\
45 \\
36\end{array}$ & $\begin{array}{l}\cdots- \\
- \\
480 \\
360 \\
288 \\
180\end{array}$ & $\begin{array}{l}600 \\
372 \\
267 \\
207 \\
166\end{array}$ \\
\hline $\begin{array}{l}\text { Druck in } \\
\text { Wasser- } \\
\text { säule } \\
\text { cm }\end{array}$ & \multicolumn{4}{|c|}{ Viskositătskoeffizienten } \\
\hline $\begin{array}{l}10 \\
20 \\
30 \\
40 \\
50 \\
60\end{array}$ & $\begin{array}{l}3,3 \\
2,8 \\
2,5 \\
2,2 \\
2,1 \\
2,1\end{array}$ & $\begin{array}{l}7,2 \\
6,5 \\
5,8 \\
5,5 \\
5,0 \\
4,7\end{array}$ & $\begin{array}{l}\overline{37,0} \\
\overline{33,4} \\
32,0 \\
23,5\end{array}$ & $\begin{array}{r}\overline{11,0} \\
10,5 \\
9,8 \\
9,4 \\
8,7\end{array}$ \\
\hline
\end{tabular}


Abhängigkeit der Viskosität von der Natur des Losungsmitte:

Der Vergleich der Viskositälen gleicn: inzentrierter Kautschuklösungen in den verschiedenen Lösungsmitteln zeigt eine große Abhängigkeit der ersteren von der chemischen Natur der letzteren. In Tab. IV sind die Durchflubzeiten resp. Viskositäts-Koeffizienren von 0,5 , ein-, zwei- und dreiprozentigen Plantagen-Kautschuklösungen $(0,5,1,2$ und $3 \mathrm{~g}$ Kautschuk iı: $100 \mathrm{ccm}$ Lösungsmittel) in den gcbräuchichen Kautschuklösungsmitteln wiedergegeben. Zur Herstellung der Lösungen wurden je $3 \mathrm{~g}$ eines dünnen Felles des erwähnten' Rohkautschuks fein zerschnitten und in $100 \mathrm{ccm}$ der verschiedenen Lösungsmittel zur Quellung und hierauf durch leichtes Schwenken in Lösung gebracht. Nach 48 stundigem Stehen im Dunkein wurde die klare Lösung von zu Boden gesunkenen unlöslichen Verunreinigungen abgegossen. Filtration durch Glaswolle er wies sich nicht als notwendig, da die filtrierten L.ösungen praktisch den gleichen Viskositätsgrad autwiesen wie die abgegossenen. Die verdünnteren Lösungen wurden aus den dreiprozentigen durch entsprechende Verdünnung unter nachheriger Kontrolle der Konzentration durch Eindampfen hergestellt.

Tabelle IV

\begin{tabular}{|c|c|c|c|c|c|c|c|c|c|}
\hline Lösungsmittel & $\begin{array}{l}\text { Durcinflue } \\
0,5 \text { Proz. }\end{array}$ & $\begin{array}{l}\text { in Seiten } \boldsymbol{c}^{\prime} \\
1 \text { Proz. }\end{array}$ & $\begin{array}{l}\text { a der Lo } \\
\left.\text { ndent }^{5}\right) \\
2 \text { Proz. }\end{array}$ & $\begin{array}{l}3 \text { Proz. } \\
\text { inngen }\end{array}$ & $\begin{array}{r}\text { Visko } \\
\text { Koeff } \\
0,5 \text { Proz. }\end{array}$ & $\begin{array}{l}\text { sitäts- } \\
\text { izienten } \\
11 \text { Proz. }\end{array}$ & $\begin{array}{l}\left(\eta_{60}=\frac{\tau_{6}^{\prime}}{x_{60}}\right. \\
2 \text { Proz. }\end{array}$ & 3 Proz. & $\begin{array}{c}\text { Aussehetı } \\
\text { der Lösung }\end{array}$ \\
\hline $\begin{array}{l}\text { Benzin } \\
\text { Benzol } \\
\text { Tetrachlorkohlenst. } \\
\text { Trichloräthylen }{ }^{6} \text {. } \\
\text { Tetracliloräthan } \\
\text { Pentachloräthan }\end{array}$ & $\begin{array}{l}13 \\
16 \\
29 \\
-38 \\
58\end{array}$ & $\begin{array}{c}29 \\
36 \\
83 \\
105 \\
166\end{array}$ & $\begin{array}{l}\overline{180} \\
- \\
- \\
\overline{883}\end{array}$ & $\begin{array}{r}630 \\
740 \\
2365 \\
508 \\
2520 \\
5460\end{array}$ & $\begin{array}{l}1,9 \\
2,1 \\
2,6 \\
2,5 \\
3,0\end{array}$ & $\begin{array}{l}4,3 \\
4,7 \\
7,5 \\
6,9 \\
8,7\end{array}$ & $\begin{array}{l}- \\
23,5 \\
- \\
\overline{46,0}\end{array}$ & $\begin{array}{r}94,0 \\
97,3 \\
211,3 \\
56,6 \\
168 \\
213,5\end{array}$ & $\begin{array}{c}\text { sehr trüb } \\
\text { vollkommen klar } \\
\text { schwach trüb } \\
\text { klar } \\
" \prime \\
" 1\end{array}$ \\
\hline
\end{tabular}

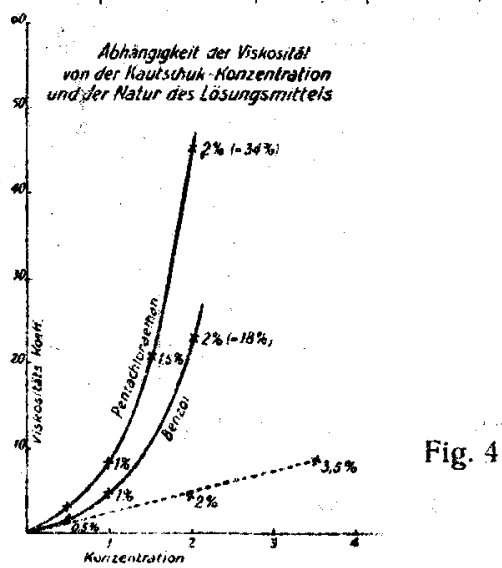

5) Es wurden die Werte beim dritten Durchgang angenommen, da diese bereits Konstanz zeigten. Sämtliche Angaben beziehen sich auf $60 \mathrm{~cm}$ Wassetsäule.

6) Diese relativ niedrige Viskosităt in Trichloräthylen dürfte auf Spuren von abgespaltener Salzsäure zurückzufuhren sein, welche die Viskosität anßBerordentlich herabdrücken. So zeigte die gleichkonzentrierte Lösung vor der Behandlung des verwendeten "Tri" mit gebranntem Kalk zur Entfernutng der (nachweisbaren) Salzsänre einen Viskositătswert von 307 Sek. resp. 34,1 Viskos.-Koeff. Vgl. auch Koll.-Zeitschr. 14, 35 (1914).

7) Diese Trubung konnte auch dureh Filtration über Glaswolle nicht entfernt werden und blieb noch nach zwei Monate langem Aufbewahren bestehen.
Es ergibt sich die interessante Tatsache, daB die Viskositäts-Koeffizienten der gleichkonzentrierten Kautschuklösungen in den chlorierten Kohlenwasserstoffen (mit Ausnahme von Trichloräthylen) ungefähr doppelt so hoch sind, als in Benzin oder Benzol (Fig. 4).

Die Ursache für dieses eigenartige Verhalten scheint nun in erster Linie auf dem verschiedenen Quellungsvermögen der verwendeten Lösungsmittel für Kautschuk zu beruhen. Nach Untersuchungen von E.Posnjak ${ }^{8}$ ) verhalten sich die im Gleichgewicht auigenommenen Flüssigkeitsvolumen von Benzol, Tetrachioräthan und Tetrachlorkohlenstoff wie 500:600:690. Nun hat E. Hatschek (loc. cit. 1) auf den EinfluB der Solvatbildung auf die Viskosität der Emulsoidsole und auf den nahen Zusammenhang der ersteren mit der Quellung hingewiesen. Er fand auf Grund theoretischer Ueberlegungen eine Beziehung zwischen der Viskosität eines Sols zum Verhälnis aus dessen Gesamtvolum zu Volumen der dispersen Phase. Berechnet man aus der von ihm gegebenen Formel $A=\left(\frac{n}{n-1}\right)^{3}$, in

8) E. Posnjak, Ueber den Queliungsdruck Kolloidchem. Beih. 3, 417 (1912). 


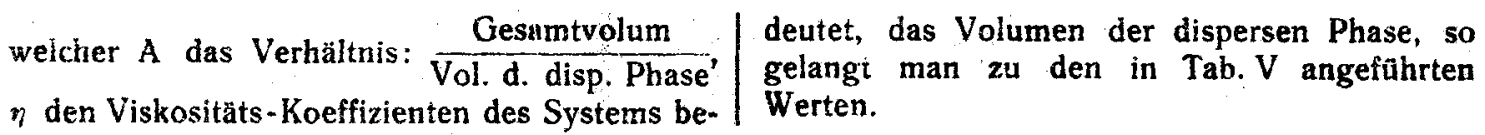

Tabelle V

\begin{tabular}{|c|c|c|c|c|c|c|c|c|}
\hline Lösungsmittel & \multicolumn{4}{|c|}{$\begin{array}{l}\text { Verh.: } \frac{\text { Gesamtvolum*) }}{\text { Vol. disp. Phase }} \\
\qquad \text { aus } A=\left(\frac{\eta}{\eta-1}\right)^{3} \\
\text { für die Konzentrationen: }\end{array}$} & \multicolumn{4}{|c|}{$\begin{array}{c}\text { Volumen (Prozente) der dispersen Phase } \\
\text { als: } \frac{\text { Gesamtvol. }}{\mathrm{A}} \\
\text { für die Konzentrationen: }\end{array}$} \\
\hline $\begin{array}{l}\text { Benzin . . . . . . } \\
\text { Benzol . . . . . } \\
\text { Tettachlorkohlenstoff } \\
\text { Tetrachlorăthan ... } \\
\text { Pentachloräthan ... }\end{array}$ & $\begin{array}{l}6,86 \\
6,1 \\
4,1 \\
4,58 \\
3,37\end{array}$ & $\begin{array}{l}2,2 \\
1,97 \\
1,44 \\
1,60 \\
1,4\end{array}$ & $\frac{\overline{1,12}}{\overline{1,06}}$ & $\begin{array}{l}1,02 \\
1,03 \\
1,00 \\
1,00 \\
1,00\end{array}$ & $\begin{array}{l}14,6 \\
16,5 \\
24,5 \\
22,0 \\
29,8\end{array}$ & $\begin{array}{l}46 \\
51,5 \\
70 \\
63 \\
72\end{array}$ & $\overline{\overline{91}}$ & $\begin{array}{l}101 \\
102 \\
103 \\
103 \\
103\end{array}$ \\
\hline
\end{tabular}

*) Es wurde angenommen, daB beim "Lösen" keine Volumănderung stattfindet (E. Posnjak).

Aus diesen Zahlen geht hervor, daB der Kautschuk in diesen dispersen Phasen ein Vielfaches seines eigenen Volumens an Lösungs-

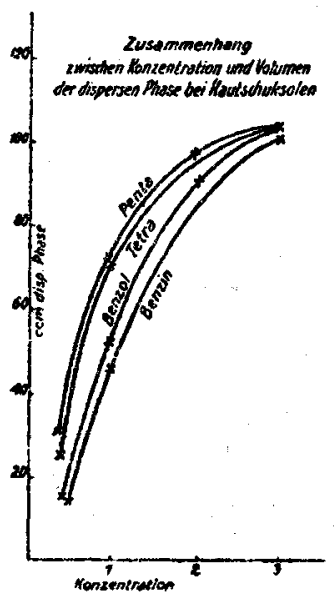

Fig. 5 mittel an sich gebunden hat. Diese Menge ist für die einzelnen Lösungsmittel verschieden und von der Konzentration des Kautschuks abhängig. (Fig. 5.)

Vergleicht man die in Lösung vom Kautschuk aufgenommenen Flüssigkeitsmengen mit dênen durch Quellung absorbierten, so ergibt sich eine bemerkenswerte Uebereinstimmung.

Diese starke Solvatation erklärt auch den abnormen Viskositătsanstieg mit geringem Anwachsen der Kautschukkonzentration. Die durch Solvatation der Lösung entzogene Flüssigkeitsmenge ist für die "Lősung" so gut wie verloren. Berechnet man aus der Differenz zwischen Gesamtvolum und Volumen der dispersen Phase die "Konzentrationen der Lösungen*, so ergeben sich Werte, welche den abnormen Viskositätsanstieg bei über ein Prozent gelöste Substanz plausibel machen. In Tab. VII finden sich dieselben zusammengestellt.

Tabelle VI

\begin{tabular}{|c|c|c|c|c|c|c|}
\hline \multicolumn{3}{|c|}{$\begin{array}{l}\text { Verhaltnis der in Lösung aufgenommenen } \\
\text { Mengen bei }\end{array}$} & \multicolumn{4}{|c|}{$\begin{array}{l}\text { Verhältnis der durch Quellung aufgenommenen } \\
\text { Mengen (n. Posnjak) bei Ueberdrucken von }\end{array}$} \\
\hline & 10,5 proz.Konz. & 1 proz. Konz. & $1120 \mathrm{~g}$ & $2120 \mathrm{~g}$ & $3120 \mathrm{~g}$ & $5120 \mathrm{~g}$ \\
\hline$\frac{\text { Tetrachlorkohlenstoff }}{\text { Benzol }}$ & 1,49 & 1,35 & 1,36 & 1,4 & 1,33 & 1,5 \\
\hline$\frac{\text { Tetrachloräthan }}{\text { Benzol }}$ & 1,33 & 1,22 & 1,12 & 1,15 & - & 1,12 \\
\hline
\end{tabular}

Lösungen von den in der Tabelle angePübrten Konzentrationen müßten mit den tatsăchlichen, die gleiche Viskositä haben, wenn bei ersteren die Solvatation der gelösten Substanz in Wegfall kãme. (Fig. 4.) 
Tabelle VII

\begin{tabular}{|c|c|c|c|}
\hline Lodsungsmittel & $\begin{array}{r}\text { Konzentration } \\
\text { berechnet aus } \\
\text { 0,5 Proz. gelöste Substanz }\end{array}$ & $\begin{array}{r}\text { ien der in Tab. V angefüh } \\
\text { gelöste Substanzm } \\
\text { samtvolum. - Volum det } \\
1 \text { Proz. gelöste Substanz }\end{array}$ & $\begin{array}{l}\text { en Lösungen } \\
\text { nge } \\
\text { spersen Phase für: } \\
2 \text { Proz. gelöste Substanz }\end{array}$ \\
\hline $\begin{array}{l}\text { Benzin } . \cdot \cdot \cdot \cdot \\
\text { Benzol : } \\
\text { Tetrachlorkohlenstoff } \\
\text { Tetrachlorathan. } \\
\text { Pentachlorathan : }\end{array}$ & $\begin{array}{l}0,58 \\
0,6 \\
0,66 \\
0,63 \\
0,70\end{array}$ & $\begin{array}{l}1,8 \\
2,0 \\
3,3 \\
2,7 \\
3,6\end{array}$ & $\frac{\overline{18}}{\overline{33}}$ \\
\hline
\end{tabular}

Unterschiede in der Viskositat verschiedener Kautschuksorten.

Aehnlich wie ein und dieselbe Kautschuksorte in verschiedenen Losungsmitteln ungleiche Viskositătswerte (bei gleicher Konzentration) aufweist, zeigen verschiedene Kautschuksorten im selben Mittel gelöst (ebenfalls gleichkonzentriert) voneinander abweichende Viskositäts- zahlen. Auf diese Erscheinung wurde bereits von Ph. Schidrowitz und H.A. Goldsbrough, G. Fol, G. Bernstein u. a. hingewiesen. Zu ihrer Erklärung dürften ähnliche Ursachen wie im früheren Falle heranzuziehen sein. So fand W. A. Caspari ${ }^{4}$, daB die Quellbarkeit verschiedener Kautschuksorten im gleichen Mittel von Sorte zu Sorte differiert.

Tabelle VIII

\begin{tabular}{|c|c|c|c|c|c|}
\hline $\begin{array}{l}\text { Kautschuk- } \\
\text { sorte }\end{array}$ & $\begin{array}{c}\text { Viskosität der } \\
\text { einprozentigen } \\
\text { Benzollösung } \\
\text { Sekunden } \\
\end{array}$ & $\begin{array}{c}\text { Viskosităts- } \\
\text { Koeffizienten } \\
\eta \\
(\text { Benzol }=1)\end{array}$ & $\begin{array}{c}\text { Viskosităt nach } \\
\text { einstündig. Er- } \\
\text { wărmen auf } 80^{\circ} \\
\text { Sekunden } \\
\end{array}$ & $\begin{array}{c}\text { Viskosităts- } \\
\text { Koeffizienten } \\
\eta^{\prime}\end{array}$ & $\begin{array}{c}\text { Aenderung in } \\
\text { Proz. durch ur- } \\
\text { sprüngliche } \\
\text { Viskos. }=100 \\
\text { Proz. }\end{array}$ \\
\hline $\begin{array}{l}\text { Para fine hard } \\
\text { Plantagen P. I } \\
\text { Plantagen P. II }\end{array}$ & $\begin{array}{r}98,6 \\
115,0 \\
63,0\end{array}$ & $\begin{array}{r}11,6 \\
15,0 \\
8,2\end{array}$ & $\begin{array}{l}68 \\
74 \\
58\end{array}$ & $\begin{array}{l}9,0 \\
9,7 \\
7,5\end{array}$ & $\begin{array}{r}22,5 \\
36,0 \\
8,5\end{array}$ \\
\hline
\end{tabular}

Anderseits haben Viskositătsmessungen desselben Forschers gezeigt, dab die in Petrolather löslichen Anteilé verschiedener Sorten bei gleicher Konzentration erhebliche Wertunterschiede aufwiesen.

Inwieweit hier der "Polymerisationsgrad" eine Rolle spielt, bleibt den Ergebnissen künftiger Untersuchungen vorbehalten. Sicher ist nur, daB die Viskositäten aller Kautschuksorten beim Erwärmen nahezu auf den gleichen Betrag zurückgehen, was auch mit den Ergebnissen von $\mathrm{G}$. Bern st e in ${ }^{10}$ ) übereinstimmt. Hierbei zeigen höherwertige Sorten begreiflicherweise eine stärkere Abnahme als minderwertige.

Da durch Erwärmung die Quellbarkeit eher erhobht wird, so scheinen die beobachteten Viskositätsabnahmen einer Depolymerisation zuzuschreiben zu sein.

Was schlieblich die Befürchtung $\mathrm{H}$ a t $\mathrm{s}$ c h e k's vom schädichen Einfluß der hohen Turbulenz auf die Viskosität der Sole im OstwaldViskosimeter betrifft, so scheint derselben keine allzugrobe Bedeutung zuzukommen. Die be- reits von G. Fol (l. c.) beobachtete Erscheinung des Viskositätsrückganges beim wiederholten Durchflieben durch die Kapillare ist nur bei konzentrierten Solen erheblicher, beträgt aber auch bei diesen nur wenige Prozente der ursprünglichen Viskosität. Da die größte Aenderung zwischen dem ersten und zweiten Durchgang zu beobachten ist, nach dem dritten aber gewöhnlich Konstanz eintritt, so scheint die anfüngliche Viskositätsabnahme $z$. T. auf die Wirkung der nichtbenetzten Gefäßwände zurückzuführen zu sein. Dies konnte auch bestätigt werden, indem eine neue Lösung in dem durch eine gleichkonzentrierte benetzten Viskosimeter beim ersten Durchgang den gleichen Wert ergab, wie die frühere Lösung bei den folgenden, und die bereits wiederholt durchs Viskosimeter getriebene Lösung bei neuerlicher

9) W. A. Caspari, Die zusammengesetzte Natur des Rohkautschuks. Journ. of the Soc. of Chem. Ind. Nr. 22 (1913). Ref. Gummi-Ztg. 28, 596 (1914).

10) G. B e rns t e in, Studien über die Vulkanisation des Kautschuks, II. Koll.-Zeitschr. 12, 193 (1913). 
Benutzung in getrockneten Instrument nahezu den gleichen Anfangswert ergab.

\section{Zusam mentasug.}

Es wurde die Beobachtung gemacht, daß3 gleichkonzentrierte Kautschuklösungen (Volum-Prozente), mit Hilfe verschiedener Lösungsmittel hergestellt, erheblich voneinander abweichende Viskositätswerte aufweisen. Als Ursache für diese Erscheinung ist in erster Linie das verschieden starke Quellungsvermögen der in Betracht kommenden Lösungs- mittel anzusehen. Diese Erklärung steht im Einklang mit von E. Posnjak gemachten Beobachtungen bei der Quellung von Rohkautschuk und mit theoretischen Ueberlegungen von E. Hatschek.

Die vorliegenden Messungen wurden mit einem Ostwald-Ubbelohde'schen KapillarViskosimeter ausgeführt, wobei die Lösungen unter einem Druck von $60 \mathrm{~cm}$ Wassersäule standen, da bei diesem die Viskositätskoeffizienten nicht zu hoch konzentrierter Lösungen vou der Durchflußgeschwindigkeit praktisch unabhängig werden.

\section{Die Kautschukchemie im Jahre 1913. (Kritischer Jahresbericht über die Fortschritte der Kautschukchemie im Jahre 1913.)}

Von Rud. Ditmar (Graz).

(Eingogangen am 17. Dez. 1913

Die Hauptarbeit auf dem Gebiete der Kantschukchemie im Jahre 1913 wurde in koristitutionsaufklärenden und kolloidchemischen Arbeiten geleistet. Die zahireich erschienenen Patente zur Herstellung von synthetischem Kautschuk stammen in ihrer Anmeldung aus früberen Jahren. Die Gegenwart ist für die praktische Verwertung der Kautschuksynthese die denkbar ungünstigste, weil der Plantagenkautschuk 1913 ganz ungeheuer im Preise gefallen ist und dabei in einer Reinheit erzielt wird, wie man sie sich nicht besser wünschen kann.

Ueber Kautschukpflanzen und Rohkautschuk sind folgende Arbeiten zu verzeichnen:

Charles P. Fox, Wilder Lattichka utschuk. (Journ. of Ind. and Engin. Chem. 5, $477-478$.)

Die beiden Kompositen Lactuca canadensis und scariola liefern einen kautschukhaltigen Latex, erstere mit 2,19 Proz. Kautschuk und 11,42 Proz. Harz, Ietztere mit 1,58 Proz. Kautschuk and 1,85 Proz. Harz. Der Kautschuk hat gute Eigenschaften.

Ceylon-Kautschuk (Bull. Inp. Inst 10, $380-383,1912$ ).

Funtumia-Kautschuk vonder Gold$k$ ü s te. (Bull. Imp. Inst. 10, 884-885, 1912.)

Chimeya-Kautschuk von Nord-WestRliodesia. (Bull. Imp. Inst. 10,385-386,1912.)

Kautschuk von Papua. (Bull. Imp. Inst. $10,386-388,1912$.
Kautschuk von Hevea Confusa aus Britisch-Guiana. (Buil. Imp. Inst. 10, 388-389, 1912.)

lmperial Institute London, Untersuchung von Parakautschukproben aus Ceylon, Indien, Südnigeria, Britisch-Guiana und Papua. (Bull. Imp. Inst. 11. Nr. 3, Juli bis September 1913.)

A. Dubosc, La Tinas, eine katschukliefernde Frucht. (Le Caoutchouc et la Guttapercha, 9, 6732; 1912.)

La Tinas ist die Frucht einer in Venezuela weitverbreiteten Liane. In ihrer äußeren Gestalt ähnelt sie einer Kaffeebohne. Beim Zerbrechen liefert sie etwas koagulierten Kautschuk. Verf: gelangte durch Extraktion der zerkleinerten Frucht mit Chloroform und Fällung der Lösung mit Azetori-Alkoholgemisch zu einem weiben, etwas klebrigen Kautschuk mittlerer Elastität, der sich rasch an der Luft schwärzt. Der Gehalt der Frucht an Kautschuk scheint zwischen 10 und 12 Proz, zu liegen.

F. Frank und E. Marckwald, Kaut. schuk-Zentralstelle fürdie Kolonien. (Ber. d. Kautschuk-Zentrale f. d. Kolonien 1912, 3. Berichtsjahr, II. Quartal, 1-21.)

24 Sorten Manihotkautschuk, die von Bäumen verschieden gedüngten Bodens stammten, wurden einer vergleichenden Untersuchung unterzogen, doch läßt sich ein zuverlässiges Bild über die Einwirkung der künstlichen Düngung bisher nach keiner Richtung hin geben. 